\title{
Surface-micromachined 2D optical scanners with optically flat single-crystalline silicon micromirrors
}

\author{
Guo-Dung J. Su, Pamela R. Patterson, and Ming C. Wu \\ Electrical Engineering Department, University of California, Los Angeles \\ Room 63-128, Eng. IV Building, 405 Hilgard Ave., Los Angeles, CA 90095
}

\begin{abstract}
We have developed a novel wafer-scale single-crystalline silicon micromirror bonding process to fabricate optically flat micromirrors on polysilicon surface-micromachined 2D scanners. The electrostatically actuated 2D scanner has a mirror area of $450 \mu \mathrm{m} \times 450 \mu \mathrm{m}$ and an optical scan angle of $\pm 7.5^{\circ}$. Compared to micromirrors made with a standard polysilicon surface-micromachining process, the radius of curvature of the micromirror has been improved by 150 times from $1.8 \mathrm{~cm}$ to $265 \mathrm{~cm}$, with surface roughness $<10 \mathrm{~nm}$. Besides, single-crystalline honeycomb micromirrors derived from silicon on insulator (SOI) have been developed to reduce the mass of the bonded mirror.
\end{abstract}

Keywords: Micromirrors, Bonding, Microelectromechanical devices, Optical scanners, Silicon on insulator, Deep reactive ion etching, Honeycomb

\section{INTRODUCTION}

MEMS (Micro-Electro-Mechanical Systems) has emerged as one of the most promising technologies to fabricate micro optical devices such as switches ${ }^{1,2}$ and scanners. ${ }^{3-5}$ High-quality scanning micromirrors are critical components of the numerous optical MEMS devices and systems. There are two main technologies used in the MEMS field: bulkmicromachining and surface-micromachining. The surface-micromachining technique is particularly attractive because of its versatility ${ }^{6}$ and its potential for integration with CMOS (Complementary Mental-Oxide-Semiconductor) circuit processes. ${ }^{7}$ However, the micromirrors fabricated by standard polysilicon surface-micromachining processes (e.g. Multi-User MEMS Process, or MUMPs ${ }^{\mathrm{TM}}$, by CRONOS) exhibit significant curvature due to residual stress of the deposited thin films. ${ }^{8,9}$ Furthermore, the surface topology is often affected by structures underneath the micromirror. ${ }^{10}$ For most applications, flat micromirrors with radius of curvature $>30 \mathrm{~cm}$ and surface roughness $<\lambda / 10$ are required. ${ }^{11}$

Though surface topology can be improved by careful layout design ${ }^{10}$ or adding a CMP process, ${ }^{12}$ the micromirrors are still subject to residual stress and stress gradient. Mirror curvature due to residual stress can be reduced by incorporating an outer edge folded frame in the mirror film ${ }^{13}$ or by exploiting the tensile stress of the mirror film to create a drum like structure on a rigid solid frame. ${ }^{14}$ Meanwhile, silicon-on-insulator (SOI) has become a popular material in bulk micromachining to fabricate MEMS devices because it simplifies the fabrication process and results in a very flat mirror surface. ${ }^{15}$

We have developed a novel hybrid bulk/surface-micromachining process to fabricate high-performance micromirrors on MUMPs chips. The micromirrors are formed on thick $(>10 \mu \mathrm{m})$ silicon-on-insulator (SOI) and then bonded to surface-micromachined actuators. This batch-fabrication process takes advantage of the flatness achievable with single crystalline silicon micromirrors without sacrificing the design flexibility of the standard polysilicon surface-micromachining process. With our integrated process, the polysilicon 2D scanner is fabricated with the planar MUMPs process while the high-quality micromirror is derived independently from the top layer of an SOI wafer ${ }^{16}$ In this paper, we describe the fabrication and performance of MEMS 2D scanners with a flat single-crystalline silicon micromirror. Optical scanners with large $(450 \mu \mathrm{m} \times 450 \mu \mathrm{m})$ and flat (radius of curvature $>265 \mathrm{~cm}$, surface roughness $<10 \mathrm{~nm}$ ) micromirrors have been successfully demonstrated. 
While this device shows orders of magnitude improvement in optical properties, the mass of the scanner is increased due to the bonded solid silicon micromirror; increased mass is undesirable in fast steering applications. To optimize the frequency response, a reduction of total mass is necessary. The honeycomb core is a well-known structural element for optimization of lightweight and strength. Micro-honeycombs have been used as molds for MEMS structure. ${ }^{17,18}$ We have expanded our integrated bonding process to include honeycomb micromirrors of single crystalline silicon. The honeycomb micromirrors are derived from SOI wafers by timed deep reactive ion etching (DRIE). Smooth mirror surface (11.5 nm) and large radius of curvature $(143 \mathrm{~cm})$ have been achieved for the honeycomb micromirror. ${ }^{19}$

\section{PRINCIPLES}

The deformation of the micromirror fabricated by standard polysilicon surface-micromachining technology is caused by residual stress and stress-gradient in the thin-film deposition processes. More insight on the factors affecting the flatness of micromirrors can be obtained by examining the analytical expression of a 1-dimension structure ${ }^{9}$ :

$$
\left.\delta\right|_{\xi=\frac{\boldsymbol{L}}{2}}=\frac{\boldsymbol{M}}{\boldsymbol{P}}\left[\sec \left(\frac{\boldsymbol{k} \boldsymbol{L}}{2}\right)-1\right], k=\sqrt{\frac{P}{E I}} \text { and } I=\int_{A} z^{2} d A
$$

where $\boldsymbol{E}$ is the Young's modulus, $\boldsymbol{I}$ is the moment of inertia, $\boldsymbol{\delta}$ is the deflection, $\boldsymbol{\xi}$ is the direction variable, $\boldsymbol{L}$ is the length of the mirror, $z$ is the mirror thickness and $\boldsymbol{A}$ is the cross-sectional area of the mirror. $\boldsymbol{P}$ is the reactive axial force and $\boldsymbol{M}$ is the bending moment, which are caused by residual stress and stress gradient, respectively. It can be found in the equation that the deflection of the mirror can be reduced by thick material (larger I). SOI is an ideal candidate for the micromirror because it is free of internal stress and has a smooth surface. A wide range of SOI material is readily available with variable silicon layer thickness, this allows for micromirror fabrication of desired thickness.

As stated previously, the mass increase of a solid mirror will affect the frequency response of the bonded 2D scanner. Single crystalline honeycombs have been designed to reduce the mass and the mass reduction afforded for typical dimensions are shown in Fig. 1.
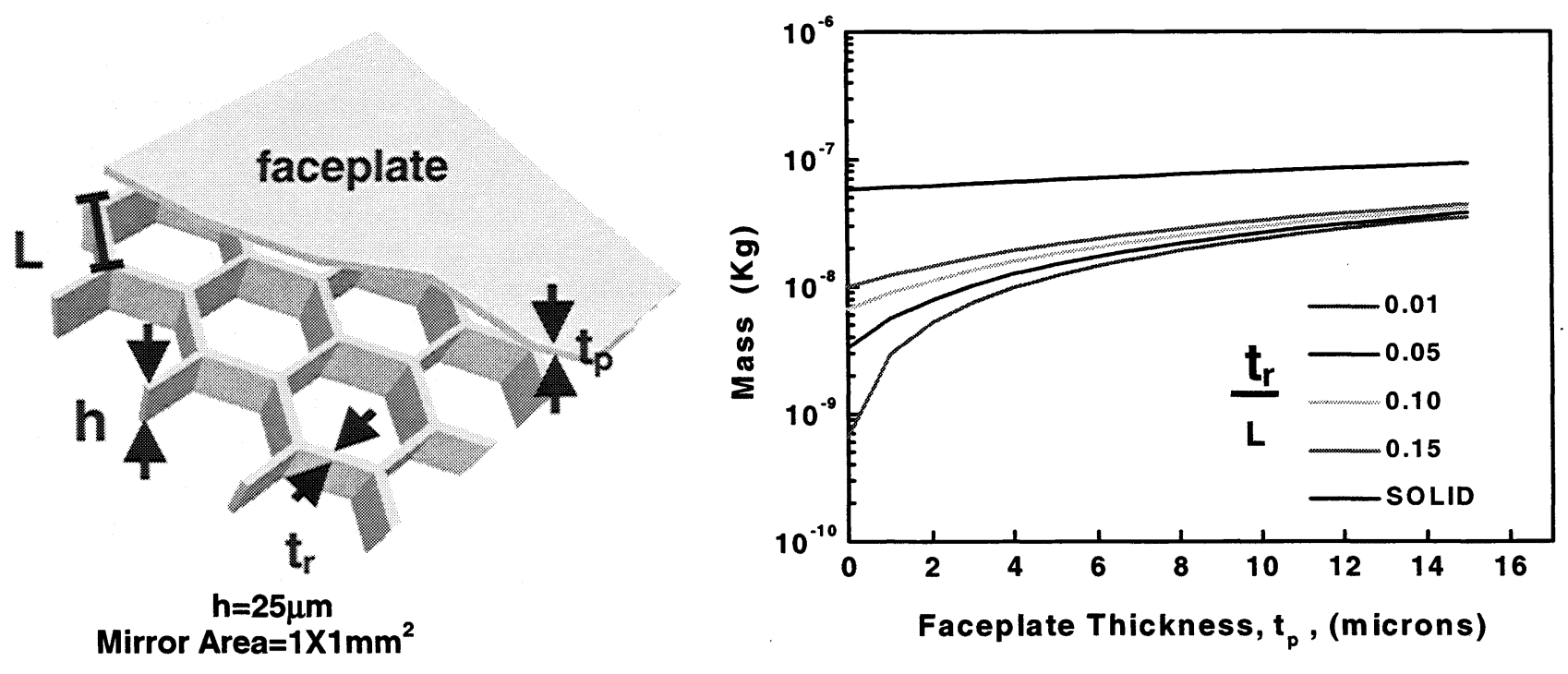

Figure 1. Honeycomb dimensions and mass reduction afforded

As can be seen, optimal weight reduction is achieved with thinner faceplates, but for thinner faceplates, $\mathrm{t}_{\mathrm{f}}<2 \mu \mathrm{m}$, a print through effect has been observed which causes a degradation of the mirror quality. The cause of this print through and the feasibility of eliminating this effect are currently under investigation. 


\section{WAFER SCALE BONDING PROCESS}

The MEMS 2D scanner used in this experiment is fabricated by the MUMPs process offered by CRONOS. The polysilicon micromirror is attached to two nested rings by orthogonal folded springs. The $2 \mathrm{D}$ scanner is realized by selfassembled MESA ${ }^{20}$ (Micro-Elevator by Self-Assembly) structures, which raise the polysilicon mirror to $>50 \mu \mathrm{m}$ above the substrate to increase the scanning angle. The self-assembly process is completely controlled by the integrated scratch drive actuators and no manual intervention is necessary. ${ }^{21}$ Scanning of the micromirror is actuated electrostatically by the four split electrodes underneath the mirror.

An SEM of the 2D scanner without and with a single-crystalline micromirror is shown in Fig. 2(a) and (b),

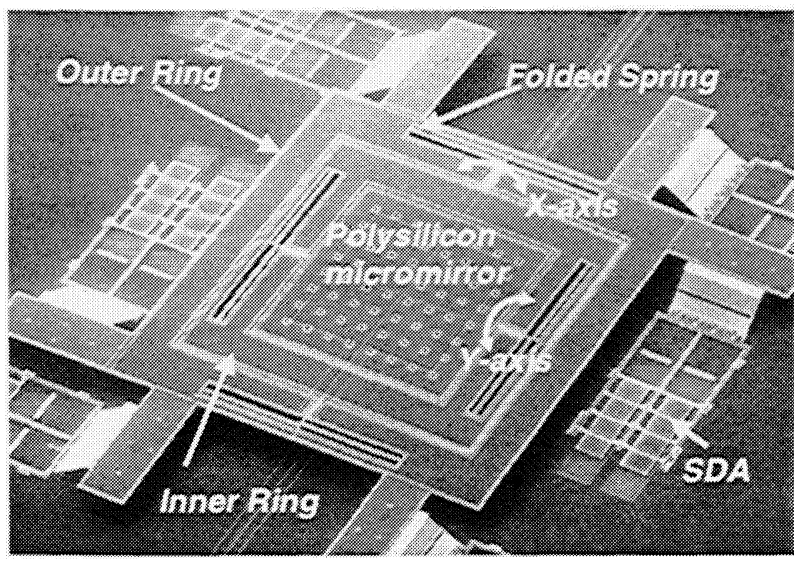

(a)

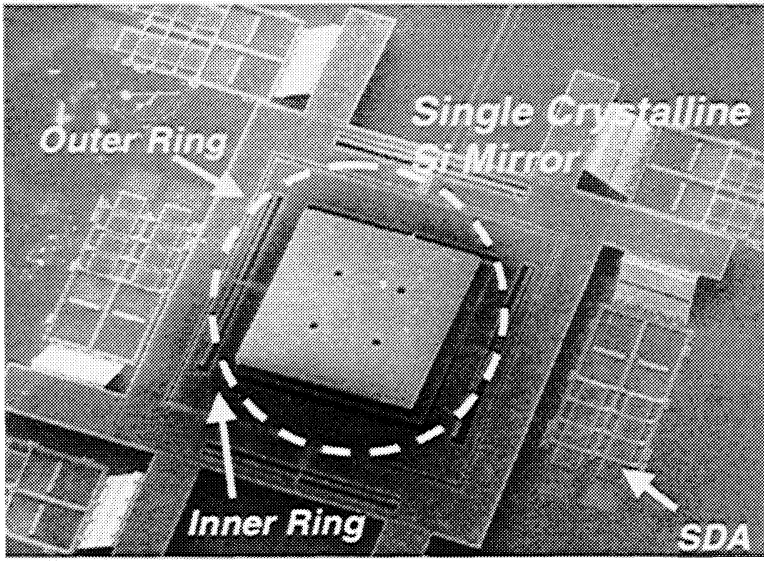

(b)

Figure 2. SEM micrographs of MEMS 2D scanner (a) without and (b) with single crystalline silicon mirror

respectively. The single-crystalline silicon mirror bonding process is described in Fig 3. First a thinned silicon-on-insulator (SOI) wafer with $23-\mu \mathrm{m}$-thick silicon layer and $200-\mu \mathrm{m}$-thick substrate is flipped over and bonded to the MUMPs chips by hard-baked photoresist (AZ 4620). The photoresist is spun over the entire chip and the bonded wafers are baked in a vacuum oven at $140^{\circ} \mathrm{C}$ for 8 hours. The SOI wafer is slightly smaller than the scanner chip so the alignment markers on the scanner chip can be utilized to align the micromirror. The substrate of the SOI wafer is then completely removed by deep reactive ion etching (DRIE), which stops on the buried oxide. The exposed area of the MUMPs chip is protected by the photoresistduring DRIE etching. The 1- $\mu \mathrm{m}$-thick buried oxide of the SOI wafer is etched away in $49 \%$ HF solution for one minute, as shown in Fig. 3(b).

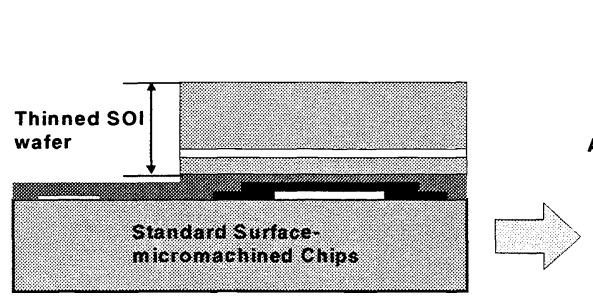

(a)

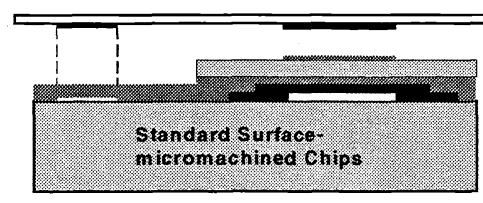

(c)

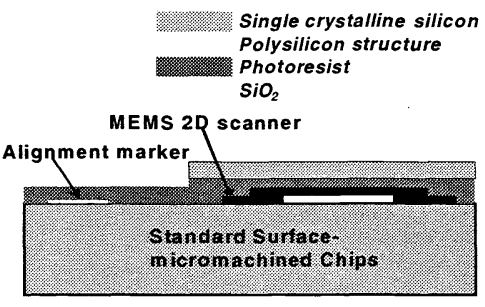

(b)
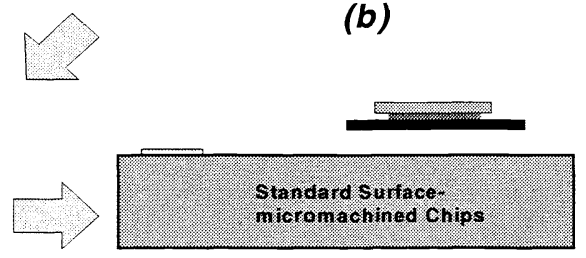

(d)

Figure 3. Schematic drawing of wafer-scale bonding process flow 
The single crystalline silicon mirror is aligned to the polysilicon actuators by photolithography and then patterned by DRIE using photoresist as a mask. After that, the remaining photoresist is cleaned by oxygen plasma and the chip is baked in an oven at $230^{\circ} \mathrm{C}$ for 3 hours to hard cure the photoresist underneath the single crystalline silicon mirror. After full release in HF solution and rinse in water and isopropanol alcohol, the MEMS 2D scanner is assembled by the on-chip actuators. The four, $15-\mu \mathrm{m}$-wide holes on the single crystalline silicon mirror facilitate the releasing process.

\section{HONEYCOMB MICROMIRRORS}

The honeycomb mirrors are formed on SOI wafers with 23- $\mu \mathrm{m}$-thick top layers. The SOI substrates are first thinned down to $200 \mu \mathrm{m}$ by mechanical lapping, as described in the wafer-scale bonding process. The honeycomb pattern (hexagonal cells with $100-\mu \mathrm{m}$-long sides and $10-\mu \mathrm{m}$-thick walls) is etched into the top surface silicon using DRIE. The etch is timed to stop before reaching the buried oxide layer such that a silicon faceplate remains. Generally speaking, the etch rate of DRIE with the standard Bosch process recipe is $\sim 2-3 \mu \mathrm{m} / \mathrm{min}$ depending upon the mask opening. After formation of the honeycomb

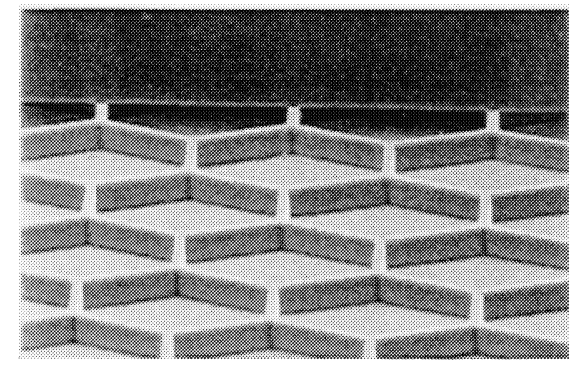

Figure 4. SEM of honeycomb structure

structure, the SOI wafer is flipped over and bonded to the actuator chip by the process described above. The 2D scanner with bonded honeycomb mirror is released and assembled as stated previously. An SEM of the underside of a honeycomb structure is shown in Fig. 4.

The etched profiles of the honeycomb structures are shown in Fig. 5 for two different etched depths. The measurements were made using an optical interferometric surface profiler, WYKO RTS 500. Honeycomb samples with faceplate thickness of $1.3,9$, and $12 \mu \mathrm{m}$ have been made. Values for average surface roughness are 123, 12, and $11 \mathrm{~nm}$ for honeycomb micromirrors with faceplate thickness of $1.3,9$, and $12 \mu \mathrm{m}$, respectively. The $123 \mathrm{~nm}$ value for the $1.3 \mu \mathrm{m}$

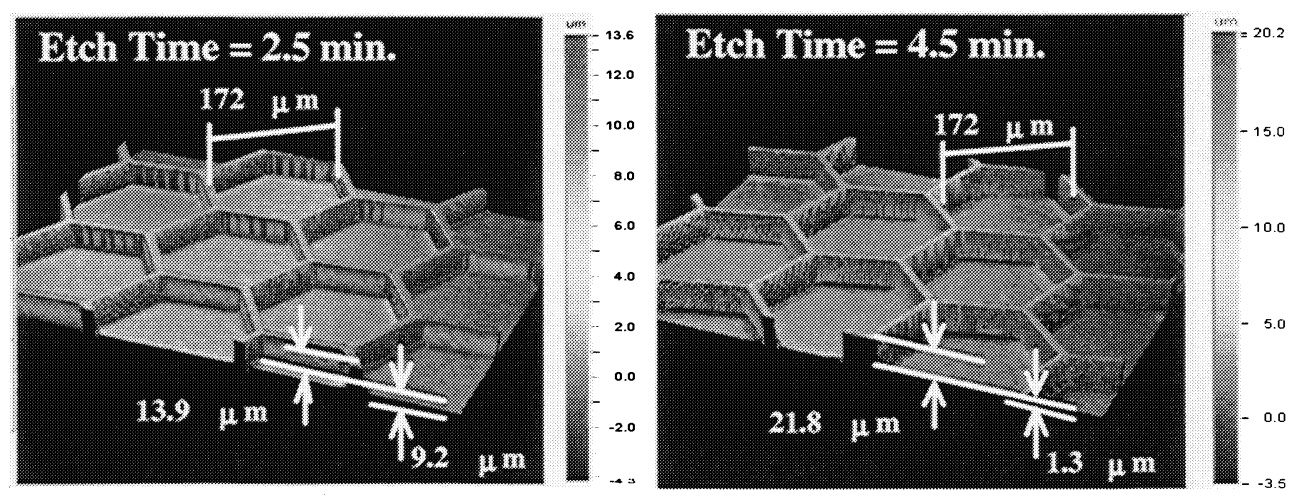

Figure 5. Honeycomb etched SOI showing depth vs. etching time

faceplate reflects the print through effect. We are currently developing a fabrication technique, which allows for precise control of the rib and faceplate thickness by using two SOI wafers and silicon fusion bonding. 


\section{EXPERIMENTAL MEASUREMENTS AND DISCUSSION}

Figure 6 shows the measured deformation of the thin-film polysilicon mirror and the single crystalline silicon mirror after they are released and assembled. Again, the measurement is performed on the WYKO RTS 500. Using the first order paraxial rays approximation, ${ }^{22}$ the radius of the curvature is calculated by the expression:

$$
R=\frac{D^{2}}{8 x}
$$

Where $R$ is radius of curvature, $D$ is the length of the mirror $(460 \mu \mathrm{m}$ in this experiment) and $\boldsymbol{x}$ is the deformation of the mirror from the center to the edge. The deformation of the polysilicon mirror is measured to be $1.45 \mu \mathrm{m}$ in convex shape, which corresponds to a curvature of $1.8 \mathrm{~cm}$. The total thickness of the polysilicon mirror is $4.25 \mu \mathrm{m}$ (Poly1 and Poly 2 with trapped Oxide2 according to MUMPs process) On the other hand, the single crystalline silicon mirror has deformation less than $10 \mathrm{~nm}$, which corresponds to a radius of curvature greater than $265 \mathrm{~cm}$. The measured thickness of the single crystalline silicon is $23 \mu \mathrm{m}$.
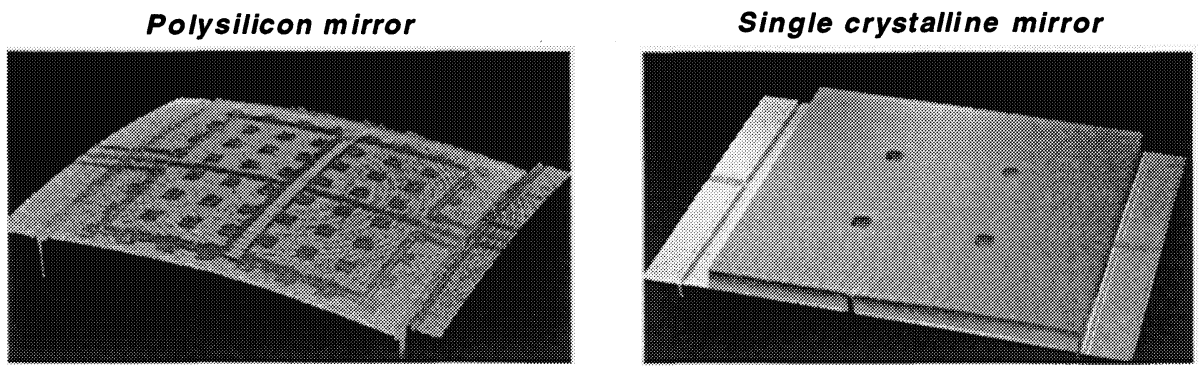

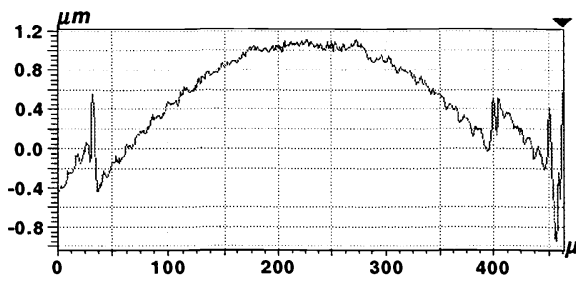

(a)

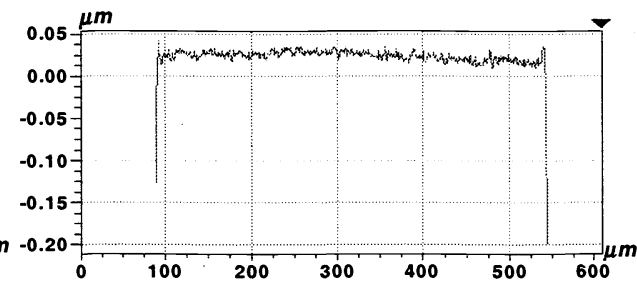

(b)

Figure 6. $3 D$ plot and cross-section profile of (a) polysilicon mirror and (b) single crystalline silicon mirror

Figure 7 shows the cross section profile and 3D plot of a bonded honeycomb mirror with a $12 \mu \mathrm{m}$ faceplate thickness. The radius of curvature for the bonded honeycomb mirror was measured to be $>140 \mathrm{~cm}$.
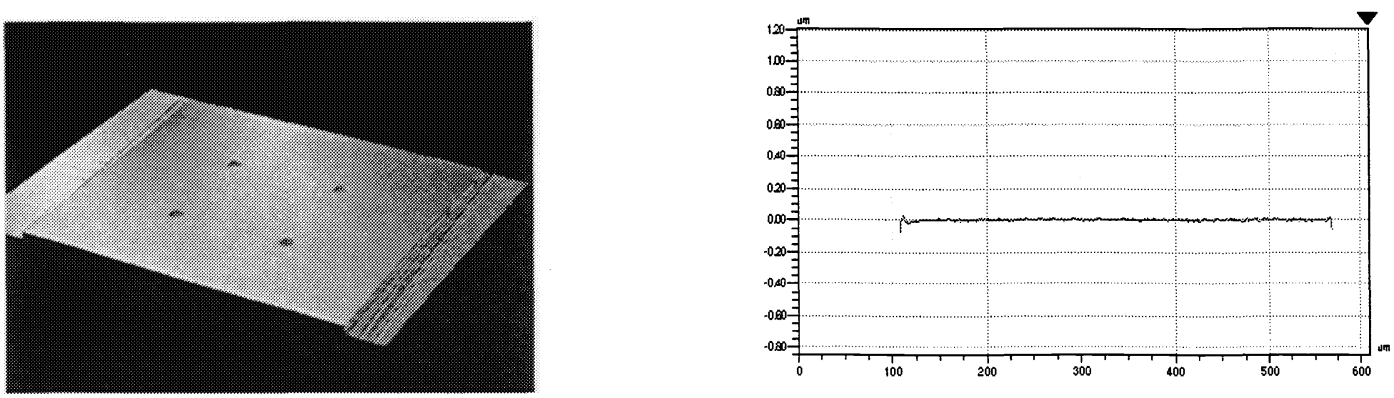

Figure 7. 3D plot and cross-section profile of bonded honeycomb mirror 


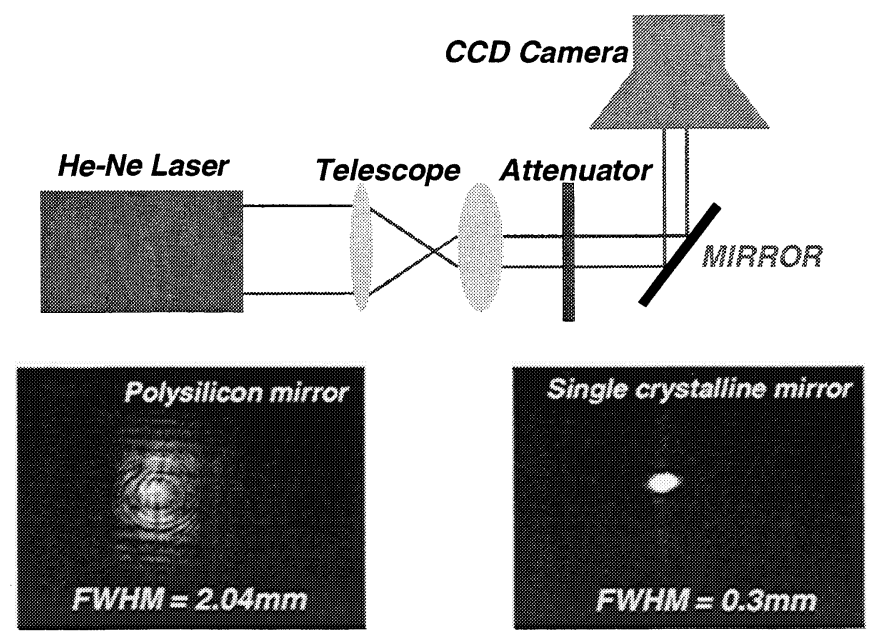

(a)

(b)

Figure 8. Experimental setup and far-field image of $(\boldsymbol{a})$ polysilicon mirror and $(\boldsymbol{b})$ singlecrystalline silicon mirror

The qualities of the micromirrors are also examined optically. The experimental setup is shown in Fig. 8. The optical beam from a He-Ne laser is reduced by a telescope to $0.28 \mathrm{~mm}$ in diameter so it is completely covered by the micromirror. A neutral density filter is used to prevent the CCD from saturating. As shown in Fig. 9, the optical quality of the single crystalline micromirror is much better than that of the polysilicon micromirror. The full-width-at-half-maximum (FWHM) spot sizes of the beams reflected from the polysilicon and the single crystalline silicon mirror are $2.0 \mathrm{~mm}$ and 0.3 $\mathrm{mm}$, respectively. That means that the maximum number of resolvable spots for the single crystalline mirror is about 7 times larger than that of the polysilicon mirror. The optical quality of the honeycomb structure surface was also examined using a

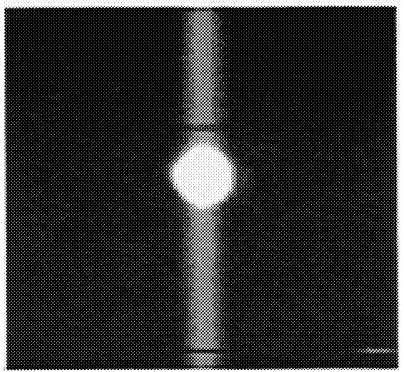

(a)

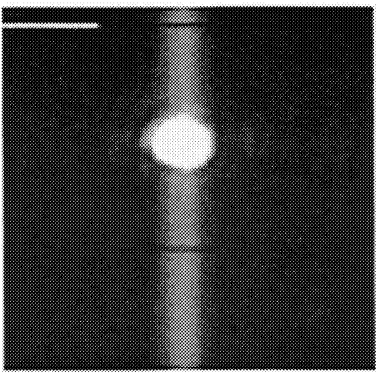

(b)

Figure 9. far-field image comparison of (a) reference silicon wafer $(\boldsymbol{b})$ honeycomb faceplate surface sample with a $9 \mu \mathrm{m}$ thick faceplate and is shown in Fig. 9. The image is essentially identical to the reference wafer.

Figure 10 shows the transfer characteristic and the frequency response of the scanner with and without a solid bonded mirror. The scanning angle versus applied voltage is almost the same for the $2 \mathrm{D}$ scanner with or without the bo nded mirror. The resonant frequencies of the scanners are at a lower frequency than was clearly resolvable on the test setup. We are currently addressing this issue in our measurement setup. The frequency response in a vacuum environment is also under investigation to determine the effect of the mass on the dynamic performance of the micromirror. 


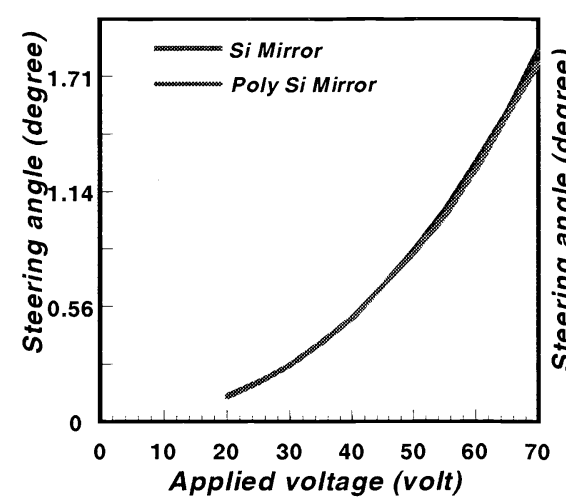

(a)

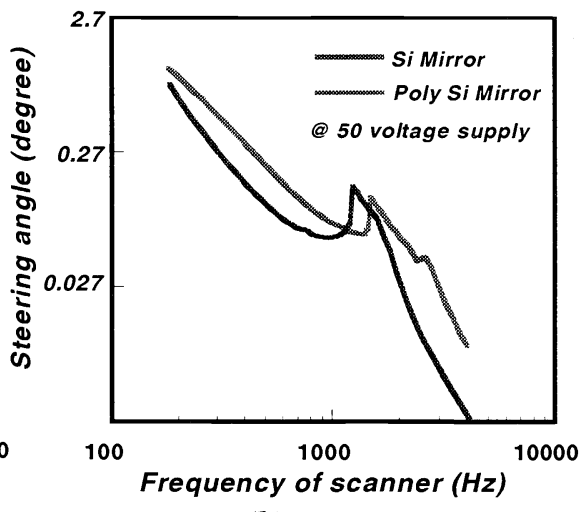

(b)

Figure 10. (a) Transfer characteristic and (b) frequency response of MEMS $2 D$ scanner

In some applications, larger mirrors are desired. Applying a similar bonding concept, we are currently modifying this novel wafer-scale bonding process to successfully bond large mirrors with $1000 \mu \mathrm{m} \times 1000 \mu \mathrm{m}$ surface area using photosensitive benzocyclobutene (BCB) as the bonding material. The modified process is more robust than our current one and will be further described in the near future.

\section{CONCLUSION}

In summary, we have successfully developed a novel wafer-scale bonding process to improve the quality of micromirrors in optical MEMS devices. MEMS 2D optical scanners with bonded single crystalline Si micromirrors have been successfully demonstrated. A radius of curvature $>265 \mathrm{~cm}$, surface roughness $<10 \mathrm{~nm}$, and maximum optical scan angle of $\pm 7.5^{\circ}$ have been achieved. In addition, the mass-reduction honeycomb mirror has been fabricated and bonded to a MEMS 2D scanner. Our new technique enables high performance Optical MEMS devices to be built using low-cost, widely accessible commercial surface-micromachining process.

\section{ACKNOWLEDGEMENTS}

The project is supported in part by DARPA under F30602-97-2-0122 and DAAH01-99-C-R220.

\section{REFERENCES}

1. R. T. Chen, H. Nguyen and M. C. Wu, "A high-speed low-voltage stress-induced micromachined $2 * 2$ optical switch," IEEE Photonics Technology Letters, vol.11, (no.11), IEEE, Nov. 1999. p.1396-8.

2. D. T. Nelson, V. A. Aksyuk, S. Arney, N. R. Basavanhally and C. R. Giles, "Fully provisioned $112 * 112$ micromechanical optical crossconnect with 35.8Tb/s demonstrated capacity," Proc. OFC 2000 PD12, Baltimore, Maryland, March 7-10, 2000.

3. L. Fan and M. C. Wu, "Two-Dimensional Optical Scanner with Large Angular Rotation Realized by Self-Assembled Micro-Elevator," Proc. IEEE LEOS Summer Topical Meeting on Optical MEMS, Paper WB4, Monterey, California, August 20-22, 1998.

4. G. J. Su, S. S. Lee and M. C. Wu, "Optical scanners realized by surface-micromachined vertical torsion mirror," IEEE Photonics Technology Letters, vol.11, (no.5), IEEE, May 1999. p.587-9.

5. M. Kiang, O. Solgaard, K. Lau and R. S. Muller, "Electrostatic combdrive-actuated micromirrors for laser-beam scanning and positioning," Journal of Microelectromechanical Systems, vol.7, (no.1), IEEE, March 1998. p.27-37. 
6. R. S. Muller and K. Lau, "Surface-micromachined microoptical elements and systems," Proceedings of the IEEE, vol.86, (no.8), IEEE, Aug. 1998. p.1705-20.

7. J. Smith, S. Montague, J. J. Sniegowski, J. R. Murray, and P. J. McWhorter, "Embedded Micromechanical Devices for the Monolithic Integration of MEMS with CMOS," IEDM Tech. Digest, pp.609-612, Dec. 1995.

8. P. M. Hagelin and O. Solgaard, "Optical raster-scanning displays based on surface-micromachined polysilicon mirrors.” IEEE Journal of Selected Topics in Quantum Electronics, vol.5, (no.1), IEEE, Jan.-Feb. 1999. p.67-74.

9. Y. Min and Y. Kim, "Modeling, design, fabrication and measurement of a single layer polysilicon micromirror with initial curvature compensation. Sensors and Actuators A (Physical), vol.A78, (no.1), Nov. 1999. p.8-17.

10. D.M. Burns and V.M. Bright, "Designs to improve polysilicon micromirror surface topology," Proceedings of the SPIE The International Society for Optical Engineering, vol.3008, (Miniaturized Systems with Micro-Optics and Micromechanics II, San Jose, CA, USA, 10-12 Feb. 1997.) SPIE-Int. Soc. Opt. Eng, 1997. p.100-10.

11. G. J. Su, W. Piyawattanmethn a, R. Rollier, L. Fan, and M. Wu "MEMS 2D scanning mirror for dynamic alignment in optical interconnect ”, Proc. Integrated Photonic Research (IPR '99), Santa Barbara, CA, July 1999.

12. R. Nasby, J. Sniegowski, J. Smith, S. Montague, C. Barron, W. Eaton, P. McWhorter, D. Hetherington, C. Apblett and J. Fleming, "Application of chemical-mechanical polishing to planarization of surface-micromachined devices." Technical Digest. Solid-State Sensor and Actuator Workshop, 1996. p.48-53

13. H-Y. Lin and W. Fang, "Torsional Mirror with an Electrostatically Drive Lever-Mechanism," Proc. IEEE/LEOS International Conference on Optical MEMS, Kauai, Hawaii, August 21-24, 2000. p.113-114.

14. J. Nee, R. Conat, M. Hart, R. Muller, K. Lau, "Stretched-film micromirrors for improved optical flatness," Proc. Of IEEE MEMS 2000, Miyazaki, Japan, Jan. 23-27, 2000, pp. 704-709.

15. K. Yamada and T. Kuriyama, " A novel asymmetric silicon micro-mirror for optical beam scanning display, "An Investigation of Micro Structures, Sensors, Actuators, Machines and Systems, Heidelberg, Germany, 25 -29 Jan. 1998, p.110-15.

16. G. J. Su, H. Nguyen, P. Patterson, H. Toshiyoshi and M.C.Wu, "Surface-Micromachined 2D Optical Scanners with High-Performance Single-Crystalline Silicon Micromirrors", (CLEO/QELS 2000), May 7-10, 2000, San Francisco, CA (Post deadline paper).

17. W.Ehrfeld,P.Bley,F.Gotz,P.Hagmann, A.Maner, J. Mohr,H.O.Moser,D.Munchmeyer,W.Schelb,D.Schmidt,E.W.Baker, "Fabrication of Microstructures using the Liga Process", Proc. IEEE Micro Robots and Teleoperators Workshop, Hyannis, MA, November 1987, pp.11/1-11/11

18. C. Keller, and M. Ferrari, "Milli-Scale Polysilicon Structures", 1994 Solid-State Sensor and Actuator Workshop, Hilton Head, pp. $132-137$.

19. P.R. Patterson, G.J. Su, H. Toshiyoshi, and M.C. Wu, "A MEMS 2-D Scanner with Bonded Single-Crystalline Honeycomb Micromirror”, Late News Digest, 2000 Solid-State Sensor and Actuator Workshop, Hilton Head, pp.17-18.

20. L. Fan, M. Wu, K. Choquette and M. Crawford, "Self-assembled microactuated XYZ stages for optical scanning and alignment", Pro. International Solid State Sensors and Actuators Conference (Transducer '97), Chicago, IL, pp. 319322.

21. T. Akiyama and H. Fujita, IEEE Micro Electro Mechanical System Workshop, Amsterdam, the Netherlands, Jan.29Feb.2, 1995

22. B. Saleh and M. Teich, "Fundamentals of Photonics," Wiley Interscience, New York, 1991. 